\title{
Improving the Culture of Safety on a High-Acuity Inpatient Child/Adolescent Psychiatric Unit by Mindfulness-Based Stress Reduction Training of Staff
}

\author{
Ilze S. Hallman, DNP, MS, PMHCNS-BC, PMHNP-BC, GNP-BC, Nancy O'Connor, PhD, ANP-BC, \\ Susan Hasenau, PhD, NNP-BC, CTN-A, and Stephanie Brady, DNP, MSN, PMHCNS-BC
}

Ilze S. Hallman, DNP, MS, PMHCNS-BC, PMHNP-BC, GNP-BC, is Clinical Nurse Specialist, Psychiatry, University of Michigan and Health Centers, Ann Arbor; Nancy O'Connor, PhD, ANP-BC, is Professor and Chair, Nursing Graduate Program, Susan Hasenau, PhD, NNP-BC, CTN-A, is Professor, Nursing Graduate Program, Madonna University, Livonia; Stephanie Brady, DNP, MSN, PMHCNS-BC, is Vice-President-Care Continuum, St. John-Providence Health System, Southfield, Michigan, USA

\section{Search terms:}

Mindfulness-based stress reduction, mental health professionals' stress, safety culture

\author{
Author contact: \\ isturis@umich.edu, with a copy to the Editor: \\ kathleen_r_delaney@rush.edu \\ The authors report no actual or potential \\ conflicts of interest. \\ No external funding was received. There was \\ internal funding.
}

doi: 10.1111/jcap.12091
PROBLEM: The purpose of this study was to reduce perceived levels of interprofessional staff stress and to improve patient and staff safety by implementing a brief mindfulness-based stress reduction (MBSR) training program on a highacuity psychiatric inpatient unit.

METHODS: A one-group repeated measure design was utilized to measure the impact of the (MBSR) training program on staff stress and safety immediately posttraining and at 2 months. Two instruments were utilized in the study: the Toronto Mindfulness Scale and the Perceived Stress Scale.

FINDINGS: The MBSR program reduced staff stress across the 2-month posttraining period and increased staff mindfulness immediately following the brief training period of 8 days, and across the 2-month post-training period. A trend toward positive impact on patient and staff safety was also seen in a decreased number of staff call-ins, decreased need for 1:1 staffing episodes, and decreased restraint use 2 months following the training period.

CONCLUSIONS: A brief MBSR training program offered to an interprofessional staff of a high-acuity inpatient adolescent psychiatric unit was effective in decreasing their stress, increasing their mindfulness, and improving staff and patient safety.

\section{Problem}

The inpatient psychiatric milieu is a stressful environment because of violence and threats that the staff is exposed to on a daily basis (Staggs, 2013). It can become dangerous on a child/ adolescent inpatient psychiatric unit if the milieu becomes chaotic, leading to unsafe practices of excessive restraint and seclusion (Cleary, Hunt, Horsfall, \& Deacon, 2012; Johnson, 2010). When all other alternatives have failed in a violent patient situation, physical restraint or seclusion is often used as a last resort. According to Delaney (1994), it is critical to have the inpatient staff "maintain the therapeutic character of the environment by controlling the milieu's tone, pace, and activity level" (Delaney, 1994, p. 5). In 2006, Delaney published a review article on restraint reduction efforts in children and adolescents. Of the five practice implications listed, the final one stated that "the content or environment of care is an important aspect of aggression management" (Delaney, 2006, p. 26).
In addition to milieu management, staff must maintain both a confident stance and calmness; including keeping their own personal anger and frustration under control. Working in a chaotic milieu can be an exhausting experience; staff needs to expend excessive energy to bring a chaotic milieu under control, and to keep patients safe. By spending their energies in this manner, they are not able to complete their more personally rewarding experiences such as assisting a patient to meet their therapeutic goals (Delaney, 1994). Hamrin, Iennaco, and Olsen (2009) completed a literature review of ecologic factors that affect inpatient psychiatric unit violence. The authors found that "nurses who are attuned to patients" fears and interactions with other patients, have an opportunity for early intervention if they observe intrusive and threatening behavior and can respond in a violencelessening way to patients (Hamrin et al., 2009, p. 218). Enhancing listening skills and presence is an important nursing intervention with a milieu and can lead to a safer environment for patients and staff. Therefore, the purpose of 
this initiative was to improve overall safety for both patients and staff by increasing nurses' ability to manage stress and increase their focus/attention on patients and the milieu.

\section{Local Situation: Model of Improvement}

In 2007, the Institute for Healthcare Improvement published a quality improvement document as part of their Innovation Series. Nolan (2007) built upon the work of Berwick (2003) and developed a simple framework to obtain improvements using a specific strategy entitled, "Will-Ideas-Execution." Berwick (2003) had defined the three aspects as having the will to improve, having alternative ideas to the status quo, and then a need to execute. The framework for execution is depicted as a triangle where the right hand side of the triangle is the framework for execution and the left hand side is the role of improvement to achieve goals and sustain change. The straight line of the triangle depicts the need to provide day-today leaders for the microsystem (Nolan, 2007, p. 5). It is important to set the performance goals first at a systems level, and then at the unit level (Nolan, 2007). Goals at the healthcare system level include successful completion of The Joint Commission visit. With regard to the adolescent inpatient unit, adhering to legal, federal guidelines for restraint use is a key systems-level performance goal.

Nelson, Batalden, and Godfrey (2007) identify clinical microsystems as a small group of people that work together consistently to provide care for a specific group of patients. For the current initiative, at the unit level, there was a focus on two dimensions: safe workplace and safe care. Safe workplace was defined as decreased absenteeism, decreased perceived staff stress levels, and increased mindfulness. Safe care was defined as a decreased number of episodes of restraint or seclusion, and a decreased number of 1:1 care episodes.

All staff members are exposed to violence and high patient acuity on a daily basis, which can be a source of stress. The nursing staff is exposed to the highest levels of violence because of the intense interpersonal contact with patients required by their care giving roles (Hamrin et al., 2009). When alternatives have failed in a violent patient situation, physical restraint, or seclusion is used (Johnson, 2010). Over a 2-month time period, prior to MBSR training, there were 30 total episodes of either restraint or seclusion on the study unit.

Although psychiatric units within general hospitals do not have patient acuity systems, such measures as number of 1:1 and 2:1 and at times 3:1 staffing ratios, can assist in defining acuity. These numbers represent the ratio of nursing staff to patients. If the situation requires restraint or seclusion, then by policy the staff mix must include at least one registered nurse (RN) for ongoing monitoring and assessment of the patient. In addition, based on the severity of the behavioral disruption, a 1:1 can be an alternative to restraint or seclusion. Two months prior to the MBSR training the number of $1: 1 \mathrm{~s}$ on the unit was 209 with $462: 1$ s. It was expected that in a safer care environment both restraint use and this number would decrease.

Having staff present to care for patients is another aspect of a safe workplace; therefore, the rate of absenteeism was used as one measure of a safe care environment. The rate of absenteeism among nursing staff 2 months prior to the MBSR training was 36, 8-hr shifts. Remaining centered and calm may assist staff to successfully approach patient care with added focus, and could impact the number of 1:1 staffing episodes. The planned intervention of MBSR training was designed to decrease the level of the staff's perceived stress and increase their ability to remain centered and calm.

Annually, the psychiatric nursing staff must complete training on the topic of safety of self and others; this federal mandate applies to all staff who work with potentially violent patients. Staff members are all on individual schedules according to their date of hire. To meet this standard, the study hospital uses the Crisis Prevention (CPI) Nonviolent Crisis Intervention ${ }^{\circledR}$ program that focuses on personal safety and includes topics such as proxemics; it does not incorporate any meditation activities. In addition to the current strategies for managing the care of violent patients, it was proposed that provision of meditation instruction and time for daily practice while staff was at work would add to their repertoire of stress management skills. This could improve the overall safety on the adolescent unit.

\section{Literature Review}

There is a long history within inpatient child and adolescent psychiatry to attempt to manage violent behaviors with restraint or seclusion. In a systematic review of seclusion and restraint use in children and adolescents, DeHert, Dirix, Demunter, and Correll (2011) found that all studies within the review had high baseline rates of restraints $(29 \%$ of patients; 43/1,000 patients) and rates of seclusion (26\% of patients; 67/1,000 patient days). The authors concluded that findings regarding the effectiveness of restraint or seclusion were absent, although there was some indication that "seclusion and restraints can lead to severe psychological and physical consequences” (p. 211). Among the authors' recommendations were to explore preventative and alternative strategies to restraint and seclusion; the MBSR training approach used in this study is seen as one such strategy.

A literature review of MBSR was completed by Praissman in 2008. Various databases were searched, including CINAHL Complete, CINAHL, Psychline, and Medline. The author concluded that MBSR is an effective treatment in the reduction of stress and anxiety. Both healthcare providers and patients that experience stress may benefit from MBSR programs. For example, in an MBSR continuing education course for healthcare providers $(2.5 \mathrm{hr}$ a week for 8 weeks, 
plus a 7-hr retreat), burnout was measured by the Maslach Burnout Inventory (MBI). Self-perceived well-being was measured by the SF-12v2. Findings demonstrated that there were significant improvements in burnout scores and mental well-being (Goodman \& Schorling, 2012).

Outcomes of MBSR have demonstrated a decrease in provider burnout and quality improvements in patient care (Brady, O'Connor, Burgermeister, \& Hanson, 2012; Cohen-Katz, Wiley, Capuano, Baker, \& Shapiro, 2005; Goodman \& Schorling, 2012; Mackenzie, Poulin, \& SeidmanCarlson, 2006). Singh et al. (2009) demonstrated a reduction in use of physical restraints and Stat medication use for destructive or aggressive behavior in clients with intellectual disabilities following a 12-week mindfulness training program for staff. Cohen-Katz et al. (2005) studied the effects of an 8-week MBSR course on nurse stress and burnout and demonstrated reduced scores on two of three MBI subscales, significantly more than waitlist controls. Changes were maintained for 3 months post-MBSR training. Penque (2009) used MBSR with staff nurses and found that it assisted them to focus on mindful thought and action patterns instead of multitasking and called for research exploring the relationship between MBSR and patient safety.

A literature review completed by Irving, Dobkin, and Park (2009) found empiric evidence that participation in MBSR programs provided benefits for clinicians' physical and mental health. As part of the critique and direction for future research, they indicated that higher attrition rates within an 8-week MBSR training may be related to the role of being a healthcare professional (Irving et al., 2009). For example, a study by Shapiro, Satin, Bishop, and Cordova (2005) experienced a $44 \%$ attrition rate in their study of MBSR in health professionals, and recommended that shorter or more efficient means of incorporating the 8-week MBSR intervention into work schedules be developed in further research. Typical dropout rates for MBSR training in nonhealthcare providers are less than 20\% (Kabat-Zinn, Lipworth, \& Burney, 1985).

Brady et al. (2012) studied the impact of MBSR training on promoting a culture of safety on an acute psychiatric unit. The program included a briefer 4-week MBSR class and demonstrated increased self-care and decreased stress levels among staff. In addition, patient safety events decreased and patient satisfaction increased following the MBSR training. Similar to Shapiro et al.'s (2005) review, the attrition rate in this 4 -week study was 30\%. Mackenzie et al. (2006) also utilized a shorter time interval of 4 weeks to provide MBSR training for a group of nurses and nurse aides. The intervention included four 30-min group sessions composed of both experiential exercises and didactic information. The 16 participants who received the intervention showed significant improvements in symptoms of burnout, enhanced relaxation, and life satisfaction compared with the 14 staff in the control group. Ando, Natsume, Kukihara, Shibata, and Ito
(2011) offered very brief MBSR training to nurses in Japan. In Session 1, participants in the experimental group $(n=15)$ were taught MBSR; they were then directed to practice at home with a CD and to return for the second session in 2 weeks to assess impact of the MBSR on their sense of coherence and well-being. Results indicated that the very brief MBSR training was effective in improving both sense of coherence and psychologic well-being when compared with a control group $(n=13)$ who received no training.

As demonstrated by several research and quality studies Ando et al. (2011), Brady et al. (2012), Mackenzie et al. (2006), and Shapiro et al. (2005), there is a need to offer "brief" MBSR training when designing the program for busy healthcare professionals because the traditional 8-week MBSR course (Kabat-Zinn et al., 1985) may not be realistic given the environment of most healthcare systems and many participants dropped out because of work demands. This project examined the ability of MBSR to increase safe care and safe work environments on a child/adolescent psychiatric unit using one of the briefest interventions currently found in the literature. There were four, 30-min MBSR classes offered over 8 days.

\section{Practice Question}

On a high-acuity inpatient child/adolescent psychiatric unit, did the provision of an intensive MBSR program improve the safety of the care environment as evidenced by an increase in mindfulness, decreased perceived stress, and decreased absenteeism?

In addition, trends on unit safety measures of restraint and seclusion episodes, and numbers of 1:1 staffing episodes were monitored before and after the MBSR training to begin to examine the potential impact of the MBSR intervention on safety of care.

\section{Methods}

The study used a one-group repeated measures design. Participants completed the Toronto Mindfulness Scale (TMS) (Lau et al., 2006) and the Perceived Stress Scale (PSS) (Cohen, Kamarck, \& Mermelstein, 1983) before the initial educational session, immediately following the 8-day training, and 2 months later.

\section{Intervention and Data Collection Procedures}

The intervention was conducted by the principal investigator who is trained in MBSR and was derived from a text on mindfulness by Williams and Penman (2011). All sessions included both didactic and experiential activities. Topics in the sessions included: waking up from autopilot, body scanning, refraining from judgment, using the breath as a stress reliever, and 
befriending yourself. Following approval of the study by the Institutional Review Board, staff volunteers were solicited by posting a signup sheet in the unit staff room. The intervention group met in four training sessions every other day for $45 \mathrm{~min}$, from 2:15 to 3:00 p.m. to maximize possible attendance between day and afternoon shifts. In addition, presession and post-session meetings were held to allow for instrument completion. Management supported adequate staffing coverage of the unit during the sessions and also allowed staff 15 min for individual meditation as part of their shift work; however, few staff actually used the 15 min allocation while at work. At the pre- and post-sessions, participants completed paper-and-pencil questionnaires, and chose their own code numbers, which were matched across data collection periods. Additionally, administrative databases were mined to extract absenteeism, seclusion, restraint, and 1:1s for 2 months prior to the MBSR intervention and again 2 months post-intervention to document emerging trends.

\section{Setting}

The study unit was a 14-bed child/adolescent psychiatric acute care unit located within a major academic medical center in southeastern Michigan. As the unit serves a tertiary level hospital, there is a high level of patient acuity. Acuity was measured by the number of 1:1s utilized per shift. Over a 2-month time period, prior to intervention, the number of 1:1s were 209 and 2:1s were 46 . The range of typical diagnoses included major depression with suicidal ideation, psychosis, and autism spectrum disorders, among others. Interprofessional interventions included education therapy, milieu therapy, psychoeducational groups, support groups, alcohol and drug education, family therapy, activity therapy, pharmacotherapy, psychotherapy and electroconvulsive therapy.

\section{Instruments}

Instruments included the PSS (Cohen et al., 1983) and the TMS (Lau et al., 2006). Demographic data were also collected. At the conclusion of the final training session, participants responded to three open-ended evaluative questions, utilized in a prior study, including (a) "What has been the value of this class to you?"; (b) "What, if any, barriers were there to your ability to practice meditation?"; and (c) "How do you feel this class will impact your work?" (Brady et al., 2012).

\section{Stress}

The PSS (Cohen et al., 1983) was utilized as a measure of staff perceived stress. This 10 -item scale asked participants to respond to the frequency of certain feelings and thoughts they have experienced in the past month. Frequencies ranged from 0 (never) to 4 (very often). Four items require reverse coding, and then scores are summed. The Cronbach's alpha coefficient has ranged from .84 to .86 (Cohen et al., 1983). Preintervention scores in the current sample ranged from 5 to 26 , with a mean of 14.83 (standard deviation $[S D]=5.7$ ) representing a moderate level of stress before the training sessions.

\section{Mindfulness}

The TMS developed by Lau et al. (2006) was used to measure mindfulness. The 13-item scale consists of 13 questions based on a Likert scale, with answers ranging from 0 (not at all) to 4 (very much). Studies have been conducted that included both individuals with meditation experience and those without. The scale demonstrates an internal consistency coefficient alpha of .88 (Lau et al., 2006). Pre-intervention scores in the current sample ranged from 13 to 47 with a mean of 28.75 $(S D=9.6)$, reflecting a moderate level of mindfulness before the training session.

\section{Findings}

\section{Data Analysis}

Data were analyzed using the Statistical Package for the Social Sciences (SPSS) version 21 software (SPSS, Inc., Chicago, IL, USA) using descriptive statistics and repeated measures analysis of variance (ANOVA). Evaluative questions were summarized by theme.

\section{Sample}

Care on the study unit is provided by an interprofessional staff including three physicians, three social workers, two school teachers, four activity therapists and 36 nursing staff. A majority of the nursing staff (RNs) and patient care workers (PCWs) work rotating 8-hr shifts, including days, evenings, and midnights. The unit employs 22 RNs, 14 PCWs, and has a full-time supervisor and part-time nurse educator who also works as a staff nurse. Participants represented both part and full-time workers. A total of 13 participants attended the initial class (15 had originally signed up). One participant attended the first session only. Twelve participants completed the four sessions and formed the study sample. Make-up sessions were held because if inclement weather and a shift containing several admissions during a scheduled session. Within the sample, the majority (10) were women and two were men. Each of the members of the interprofessional team was represented within the sample. The majority (6) were registered nurses, two were teachers, and there was one each including PCW, social worker, activity therapist, and physician. Most participants were highly experienced workers, with a mean 
length of time working on the unit of 11 years with a range from 8 months to 32 years. Participants ranged in age from 23 to 56 years, with a mean age of 39 years. Four participants were aware of MBSR, and four participants practiced a form of meditation (33\%) prior to the study; however, none of the participants had extensive experience with MBSR. The majority of the sample worked the day shift (9), 1 worked the midnight shift, and 2 worked rotating shifts.

\section{Results}

The study examined whether the provision of a brief MBSR training session for staff would improve the safety of care for adolescent psychiatric inpatients and improve the safety of the work environment. For the question of whether the training improved the safety of the work environment, three measures of safe work environment were examined. Absenteeism during the 2 months prior to the intervention was $288 \mathrm{hr}$ or 36 shifts, whereas during the 2 months post-MBSR intervention the rate was $248 \mathrm{hr}$ or 31 shifts, a decline of five shifts for the 2-month period.

A one-way repeated ANOVA (Table 1) was conducted to compare scores on the PSS at three times: pre, post, and 2 months after the intervention. There was a significant effect over time, Wilks' lambda $=.35, F(2,10)=9.246, p<.05$. A decrease in the PSS signifies a decrease in stress.

A one-way repeated ANOVA (Table 1) demonstrated that differences in the TMS score at different stages of the study were statistically significant. The TMS scores increased significantly from the pretest $(M=28.75, S D=9.6)$ to post-test $(M=35.08, S D=8.34)$. The TMS score increased after 2 months $(M=36.25, S D=9.6)$. Wilkes' lambda $=.456$, $F(2,10)=5.958, p<.05$. Higher scores on the TMS denote increased mindfulness.

\section{Open-Ended Question Evaluation Themes}

A thematic analysis was conducted with the content from the training evaluation questions. The intervention was rated positively by all 12 participants. Themes of the value of the intervention to the staff included enhanced interventions with patients as well as an enhanced quality of life for staff. Statements included: (a) "hoping for a 'quick' way to de-stress-especially when dealing with difficult patients,"

Table 1. Safe Care Environment Pre- and Post-MBSR Intervention

\begin{tabular}{llll}
\hline & Pre-MBSR & Post-MBSR & $\begin{array}{l}\text { Two months } \\
\text { post-MBSR } \\
\text { Type of measure }\end{array}$ \\
$M(S D)$ & $M(S D)$ & $M(S D)$ \\
\hline Perceived Stress Scale & $14.83(5.72)$ & $13.67(5.45)$ & $9.67(4.23)$ \\
Toronto Mindfulness Scale & $28.75(9.54)$ & $35.08(8.4)$ & $36.42(9.6)$ \\
\hline
\end{tabular}

MBSR = mindfulness-based stress reduction; SD = standard deviation. and (b) "furthering mindfulness practice, I believe, can potentially improve the quality of care I offer and perhaps the quality of my life."

Identified barriers to the ability to practice meditation included: (a) "sometimes just getting caught up in the many events in life-kids, work, school, etc.," and (b) "finding time to put it into my routine, however as I kept at the meditations, I found I was practicing mindfulness throughout the day." Staff commented here on not really using the homework logs to document their home practice.

Nine out of 12 participants offered additional comments related to the impact of the MBSR training on their work. A sample of these responses include: (a) "being more relaxed and present with the patients and families;" (b) "a simple exercise can change patient/staff interactions and hopefully make patient contact more meaningful for the patients;" (c) "a quick way to prepare/center self when needing to approach anticipated stressful situation/interaction;" (d) "I feel that it will make me more relaxed around patients and staff. I can deal with changes more efficiently and handle things that are outside of my control;" (e) "I believe it can improve my focus and attention to process and to patient. I can be more effective as a clinician when I can remain 'in the moment"'; and (f) "Improve ability to stay mindful and avoid judgment when working with patient and families."

\section{Discussion}

A brief MBSR training program offered to an interprofessional staff of a high-acuity inpatient adolescent psychiatric unit was effective in decreasing their stress, increasing their mindfulness, reducing staff absenteeism, and improving staff safety. Patient safety trended upward on the unit following the MBSR training; the number of restraints and seclusions decreased from the pre-intervention period (30 episodes in 8 weeks) to the post-intervention period (10 episodes in the 8 weeks following the training). Additionally, the number of 1:1 care episodes also decreased from preintervention (209 in an 8-week period) to post-intervention (183 in 8 weeks). Strikingly, the number of 2:1 staff episodes also decreased from a pre-intervention number of 46 in the weeks to zero in the 8 -week post-intervention period.

The shorter time of intervention ( 8 days versus $4-8$ weeks) may have accounted for the retention of the study participants across the 2-month period. The interprofessional nature of the participants potentially strengthened the intervention to enable more of an impact on the unit's milieu. Many of the participants were informal or formal leaders of the unit. Monitoring numbers of restraints and seclusion on a monthly basis, as well as proxy measures of acuity such as 1:1 staffing episodes and reporting these numbers to staff, as well as to external regulators may help to establish a culture of safer care on the unit. In addition, acknowledging the goal of 
creating a safer work environment by reducing staff stress levels and increasing their mindfulness via investment in staff development such as the brief MBSR training program developed herein can help to develop a culture of safety on inpatient adolescent psychiatric units. The majority of participants when asked "How do you feel this class will impact your work?" identified feeling more present and less judgmental and also viewed MBSR as a strategy to utilize when approaching a potentially stressful situation/interaction. They also identified awareness of "being in the moment" at the work place, which is an important safety factor when working in a highly acute inpatient child/adolescent psychiatric unit.

\section{Limitations}

A limitation of this study was its small sample size and its self-selection by study participants. It is not known whether these same results would be attained with a larger and more heterogeneous sample of staff members who were less experienced than the current sample. These factors limit the generalizability of the findings. Nonetheless, many staff participants are thought leaders and early adopters to change on the unit; therefore, despite only a third of the staff being trained to date in MBSR, their influence can be seen in the initial positive trends noted on key safety measures.

In addition, self-report was required for all study measures, and is subject to response bias. In this study, there were not interventions with the participants after the initial four MBSR sessions. While homework logs and 15 min of practice time were offered as additional incentives, few actually used them, resulting in an even simpler and less costly staff training program. A recommendation for the future is to provide weekly emails or drop in sessions to keep the meditation activities at the forefront of the safe care delivery process, which may enhance further improvements in stress levels, mindfulness, and safety measures.

\section{References}

Ando, M., Natsume, T., Kukihara, H., Shibata, H., \& Ito, S. (2011). Efficacy of mindfulness-based meditation therapy on the sense of coherence and mental health of nurses. Health, 3(2), 118-122.

Berwick, D. M. (2003). Errors today and errors tomorrow. New England Journal of Medicine, 34, 2570-2572.

Brady, S., O'Connor, N., Burgermeister, D., \& Hanson, P. (2012). The impact of mindfulness meditation in promoting a culture of safety on an acute psychiatric unit. Perspectives in Psychiatric Care, 48(3), 129-137.

Cleary, M., Hunt, G. E., Horsfall, J., \& Deacon, M. (2012). Nurse-patient interaction in acute adult inpatient mental health units: A review and synthesis of qualitative studies. Issues in Mental Health Nursing, 33, 66-79.
Cohen, S., Kamarck, T., \& Mermelstein, R. (1983). A global measure of perceived stress. Journal of Health and Social Behavior, 24, 386-396.

Cohen-Katz, J., Wiley, S. D., Capuano, T., Baker, D. M., \& Shapiro, S. (2005). The effects of mindfulness-based stress reduction on nurse stress and burnout. Part II: A quantitative and qualitative study. Holistic Nursing Practice, 19(1), 26-35.

DeHert, M., Dirix, N., Demunter, H., \& Correll, C. (2011). Prevalence and correlates of seclusion and restraint use in children and adolescents: A systematic review. European Child Adolescent Psychiatry, 20, 221-230.

Delaney, K. R. (1994). Calming an escalated milieu. Journal of Child and Adolescent Psychiatric Nursing, 7(3), 5-13.

Delaney, K. R. (2006). Evidence base for practice: Reduction of restraint and seclusion use during child and adolescent psychiatric inpatient treatment. Worldviews on Evidence-based Nursing, 3(1), 19-30.

Goodman, M. J., \& Schorling, J. B. (2012). A mindfulness course decreases burnout and improves well-being among healthcare providers. International Journal of Psychiatry in Medicine, 43(2), 119-128.

Hamrin, V., Iennaco, J., \& Olsen, D. (2009). A review of ecological factors affecting inpatient psychiatric unit violence: Implications for relational and unit cultural improvements. Issues in Mental Health Nursing, 30, 214-226.

Irving, J. A., Dobkin, P., \& Park, J. (2009). Cultivating mindfulness in health care professionals: A review of empirical studies of mindfulness-based stress reduction (MBSR). Complementary Therapies in Clinical Practice, 15, 61-66.

Johnson, M. E. (2010). Violence and restraint reduction efforts on inpatient psychiatric units. Issues in Mental Health Nursing, 31, 181-197.

Kabat-Zinn, J., Lipworth, L., \& Burney, R. (1985). The clinical use of mindfulness meditation for the self-regulation of chronic pain. Journal of Behavioral Medicine, 8(2), 163-190.

Lau, M. A., Bishop, S. R., Segal, Z. V., Buis, T., Anderson, N. D., Carlson, L., . . Carmody, J. (2006). The Toronto mindfulness scale: Development and validation. Journal of Clinical Psychology, 62(12), 1445-1467.

Mackenzie, C. S., Poulin, P. A., \& Seidman-Carlson, R. (2006). A brief mindfulness-based stress reduction intervention for nurses and nurse aides. Applied Nursing Research, 19(2), 105-109.

Nelson, E., Batalden, P., \& Godfrey, M. (2007). Quality by design: A clinical microsystem approach. San Francisco: Jossey-Bass Publishers.

Nolan, T. W. (2007). Execution of strategic improvement initiatives to produce system-level results. IHI Innovation Series white paper. Cambridge, MA: Institute for Healthcare Improvement.

Penque, S. (2009). Mindfulness based stress reduction effects on registered nurses. Retrieved from http://dissexpress.umi.com/dxweb/search.html.

Praissman, S. (2008). Mindfulness-based stress reduction: A literature review and clinician's guide. Journal of the American Academy of Nurse Practitioners, 20(4), 212-216. 
Shapiro, S. L., Satin, J. A., Bishop, S. R., \& Cordova, M. (2005). Mindfulness-based stress reduction for health care professionals: Results from a randomized trial. International Journal of Stress Management, 12(2), 164-176.

Singh, N., Lancioni, G., Winton, A., Singh, A., Adkins, A., \& Singh, J. (2009). Mindful staff can reduce the use of physical restraints when providing care to individuals with intellectual disabilities.
Journal of Applied Research in Intellectual Disabilities, 22, 194-202.

Staggs, V. S. (2013). Nurse staffing, RN mix, and assault rates on psychiatric units. Research in Nursing \& Health, 36(1), 26-37.

Williams, M., \& Penman, D. (2011). Mindfulness: An eight week plan for finding peace in a frantic world. New York: Rodale Press. 